\title{
Fortificación de la harina de trigo con ácido fólico: cambios en la tasa de defectos del tubo neural en Chile (1)
}

\author{
Cortés F, Mellado C, Pardo RA, Villarroel LA, Hertrampf E. Wheat flour fortifica- \\ tion with folic acid: changes in neural tube defects rates in Chile. Am J Med Genet $A$ \\ 2012;158A(8):1885-90
}

Análisis crítico: Constanza Ralph, Jorge A. Carvajal (PhD). División de Obstetricia y Ginecología, Unidad de Medicina Materno-Fetal, Facultad de Medicina, Pontificia Universidad Católica de Chile.

\section{RESUMEN}

En enero del 2000, el Ministerio de Salud de Chile ordenó la adición de ácido fólico (AF) a la harina para reducir el riesgo de defectos del tubo neural (DTN). Esta política resultó en un aumento significativo en el nivel de folato sérico y en los glóbulos rojos en las mujeres en edad fértil un año después de la fortificación. Para evaluar el efecto de la fortificación de la harina en la prevalencia de DTN en Chile, diseñamos un programa de vigilancia hospitalaria para monitorizar la frecuencia de DTN en todos los partos (vivos y mortinatos) con peso $\geq 500$ gramos en nueve maternidades de hospitales públicos de Santiago, Chile, desde 1999 al 2009. Durante el período pre-fortificación (1999-2000) la tasa de DTN fue $17,1 / 10.000$ nacimientos en un total de 120.566 recién nacidos. Durante el período post-fortificación (2001-2009) la tasa de DTN disminuyó a 8,5/10.000 nacimientos, en un total de 489.915 recién nacidos, lo que se traduce en una tasa de reducción de $50 \%$ (RR: 0,5; IC95\%: 0,42-0,59) para todos los DTN. La tasa de reducción por tipo de DTN estudiado fue: $50 \%$ en anencefalia (RR: 0,5; IC95\%: 0,38-0,67), 42\% en encefalocele (RR: 0,58; IC95\%: 0,37-0,89) y 52\% en espina bífida (RR: 0,48; IC95\%: 0,38-0,6). Las tasas muestran una significativa reducción en mortinatos y en nacidos vivos: 510,3 a 183,6/10.000 $(R R=0,36$; IC95\%: 0,25-0,53) y 13,3 a 7,5/10.000 ( $R R=0,56$; IC95\%: 0,47-0,68) respectivamente. En Chile, la fortificación de la harina con AF ha probado ser una estrategia efectiva para la prevención primaria de DTN.

\section{ANÁLISIS DE LA INVESTIGACIÓN}

\section{A. Relevancia de la investigación}

Los defectos del tubo neural son la segunda causa de malformaciones fetales después de los defectos cardiacos, con alta tasa de morbimortalidad asociada. Representan la principal malformación congénita prevenible gracias a cambios nutricionales de la salud pública $(2,3)$. El riesgo de tener un DTN puede reducirse en $50-70 \%$ por la suplementación periconcepcional con ácido fólico (2). Recomendaciones internacionales sugieren a toda mujer en edad fértil sin factores de riesgo un consumo diario de 400 ug de ácido fólico para reducir el riesgo de DTN (4), y esto se considera un pilar del consejo pre-concepcional en nuestros días. Dado que en Chile existe poco control preconcepcional del embarazo, principalmente en el sector de menos recursos, se hace necesario una medida de prevención que logre suplementar incluso a aquellas mujeres que no planifican su embarazo o inician su control tardíamente. En Chile, desde el año 2000 se incorporó ácido fólico a la harina de trigo, con la intención de reducir los defectos del tubo neural. Este artículo revisa el resultado de esta intervención nutricional.

\section{B. El estudio (1)}

Diseño: Estudio de vigilancia prospectiva que compara la prevalencia de DTN en mujeres chilenas atendidas en la maternidad de 9 hospitales públicos de Santiago, Chile, en el periodo pre-fortificación 
Tabla I

RESULTADOS DEL ESTUDIO

\begin{tabular}{lcccccc}
\hline & $\begin{array}{c}\text { Pre-fortificación } \\
\mathrm{n}\end{array}$ & $\begin{array}{c}\text { 1999-2000 } \\
\text { Tasa/10000 }\end{array}$ & $\begin{array}{c}\text { Post-fortificación } \\
\mathrm{n}\end{array}$ & $\begin{array}{c}\text { 2000-2009 } \\
\text { Tasa/10000 }\end{array}$ & RR & IC 95\% \\
\hline Anencefalia & 72 & 6,0 & 147 & 3,0 & 0,50 & $0,38-0,67$ \\
Encefalocele & 29 & 2,4 & 68 & 1,4 & 0,58 & $0,37-0,89$ \\
Espina bífida & 105 & 8,7 & 204 & 4,2 & 0,48 & $0,38-0,6$ \\
Total DTN & 206 & 17,1 & 419 & 8,6 & 0,50 & $0,42-0,59$ \\
\hline
\end{tabular}

con ácido fólico (1999-2000) y post-fortificación con ácido fólico (2001-2009). Estos hospitales eran parte del Sistema de Salud Pública del país, lugar donde se atiende la población de bajos recursos.

Recolección de datos: En cada uno de los hospitales hubo un médico o matrona entrenada que diariamente revisó los registros de partos para consignar a los recién nacidos (RN) con defectos del tubo neural. La información primaria fue revisada por los autores mediante el registro de RN con malformaciones congénitas, registro de RN hospitalizados, reportes de alta hospitalaria, registro de mortinatos, auditorías de las muertes fetales, registro de muertes en menores de un año, protocolos de autopsia y registros clínicos. Pacientes: RN o mortinatos con DTN cuyo peso fuese $\geq 500 \mathrm{~g}$, nacidos en el periodo descrito. Los DTN evaluados fueron anencefalia, encefalocele y espina bífida; en forma aislada o asociado a otra malformación. Resultados rescatados de datos anotados por encargado del proyecto en cada centro. Espina bífida oculta o DTN producido por banda amniótica fueron excluidos del estudio. Resultados: La tasa de DTN disminuyó en $50 \%$ en el periodo estudiado.La Tabla I muestra la disminución significativa de casos de anencefalia, encefalocele y espina bífida específicamente.

\section{Análisis crítico}

Estudio de buena calidad, nivel de evidencia tipo II-b. Un estudio randomizado controlado si bien habría sido ideal, nos parece poco plausible, pues implicaría impedir el consumo de harina fortificada en el grupo control. Respecto de la metodología usada, destaca la buena calidad del estudio en relación a la recolección de datos. Estos fueron adquiridos agotando todas las fuentes de registros, lo cual hace que los resultados sean confiables. Podría discutirse si las poblaciones estudiadas (pre-fortificación vs. post-fortificación) son comparables y solo diferentes en la intervención estudiada. Pensamos que si son com- parables, pues se trata de hospitales públicos de Santiago, que representan a población de bajos recursos, con poca movilidad, y poco acceso a medicina privada. Pese a la popularidad del uso preconcepcional del ácido fólico en 1999-2000, es poco probable que esta población lo usara. Es posible postular que la reducción en defectos del tubo neural al nacimiento se debe al diagnóstico ecográfico precoz y aborto eugenésico, lo que requeriría revisar el grado de acceso a ecografía en los dos períodos estudiados. Si esta posibilidad hubiese sido cierta, habríamos esperado una mayor reducción en la tasa de anencefalia, siendo esta una malformación de fácil diagnóstico y muy precoz en el embarazo. Sin embargo, la tasa de encefalocele y espina bífida, cuyo diagnóstico es de mayor dificultad en el primer trimestre, se redujo en la misma proporción. Este argumento, además de la ilegalidad del aborto en Chile, descarta esta hipótesis. Nos parece que el estudio es de buen diseño, sin fuentes evidentes de sesgo (en su nivel de evidencia), y con un resultado clínicamente muy relevante. La información obtenida es original y contributaria.

\section{CONCLUSIONES}

Estudio de buena calidad, con resultados de gran importancia para justificar la práctica actual de fortificación de la harina con ácido fólico en nuestro país. Deja la puerta abierta a la discusión sobre la necesidad de suplementación pre-concepcional con ácido fólico (adicional al de la harina) en Chile.

\section{REFERENCIAS}

1. Cortes F, Mellado C, Pardo RA, Villarroel LA, Hertrampf $E$. Wheat flour fortification with folic acid: changes in neural tube defects rates in Chile. Am J Med Genet A 2012;158A(8):1885-90.

2. De-Regil LM, Fernández-Gaxiola AC, Dowswell $T$, Peña-Rosas JP. Effects and safety of periconceptional 
folate supplementation for preventing birth defects. Cochrane Database Syst Rev. 2010 Oct 6;(10):CD007950.

3. Nazer J, López-Camelo J, Castilla EE. [ECLAMC: 30year study of epidemiological surveillance of neural tube defects in Chile and Latin America]. Rev Med Chil
2001;129(5):531-9.

4. Center for disease control and prevention. Recommendations for the use of folic acid to reduce the number of cases of spina bifida and other neural tube defects. MMWR Recomm Rep 1992;41(RR-14):1-7 